\title{
Changes in Cerebral Blood Flow Detected by SPECT in Type 1 and Type 2 Diabetic Patients
}

\author{
Miklós Káplár ${ }^{1}$, György Paragh ${ }^{1}$, Annamária Erdei ${ }^{1}$, Éva Csongrádi ${ }^{1}$, Éva Varga ${ }^{1}$, Ildikó Garai ${ }^{2}$, Lajos Szabados ${ }^{2}$, \\ László Galuska² , and József Varga² \\ ${ }^{1} 1$ st Department of Internal Medicine, University of Debrecen Medical and Health Science Center, Debrecen, Hungary; and \\ ${ }^{2}$ Department of Nuclear Medicine, University of Debrecen Medical and Health Science Center, Debrecen, Hungary
}

\begin{abstract}
Although macrovascular complications are typical for type 2 diabetes mellitus (T2DM), cerebral microvascular damage develops both in type 1 diabetes mellitus (T1DM) and T2DM. Color Doppler ultrasound is widely used for the examination of large- and medium-sized arteries, whereas SPECT and MRI are capable of identifying disturbances in the circulation of microvessels. Former studies using semiquantitative methods showed reduced reactivity and reserve capacity of cerebral vessels in both T1DM and T2DM patients. Our aim was to investigate whether there was any difference in the effects of the 2 types of diabetes mellitus on the global or regional cerebral blood flow, influenced by microvascular damage. Methods: In our study, the circulation and reserve capacity of cerebral arteries was examined using 99mTc-hexamethylpropylene amine oxime SPECT. A total of 17 individuals with T1DM and 43 individuals with T2DM were involved in the study. Results: Both basal and acetazolamide-challenged brain circulation were significantly lower in T2DM patients than in T1DM patients. We did not find a significant difference in the reserve capacity. However, the circulation of the frontal and occipital lobes changed differently in the 2 groups. The ratio of the circulation of the frontal and occipital lobes was significantly reduced both in basal and in acetazolamide-stimulated states in T2DM patients, independently of age $(P<0.0005$ and $P<0.017)$, showing a greater relative decrease in the circulation of the frontal lobe in T2DM patients. Conclusion: There was a significant association between basal brain circulation and age, body mass index, and high-density lipoprotein (HDL), whereas acetazolamide-stimulated circulation showed a significant association with serum triglyceride and HDL.
\end{abstract}

Key Words: diabetes mellitus; cerebral perfusion; SPECT; reserve capacity; frontooccipital ratio

J Nucl Med 2009; 50:1993-1998

DOI: 10.2967/jnumed.109.066068

D iabetes mellitus considerably increases the risk of stroke due to the damage of cerebral arteries $(1,2)$. Macrovascular complications are typical for type 2 diabetes

Received May 21, 2009; revision accepted Aug. 17, 2009.

For correspondence or reprints contact: Miklós Káplár, University of Debrecen Medical and Health Science Center, 1st Department of Internal

Medicine, H-4012 Debrecen, Nagyerdei krt. 98, Hungary.

E-mail: mkaplar@internal.med.unideb.hu

COPYRIGHT () 2009 by the Society of Nuclear Medicine, Inc. mellitus (T2DM); however, the injury of cerebral microvessels can be detected in both type 1 diabetes mellitus (T1DM) and T2DM. Hyperglycemia leads to structural damage of capillaries and endothelial dysfunction, and the permeability of the blood-brain barrier is also altered in diabetics (3). These factors significantly affect the regional and global cerebral blood flow (CBF) and the metabolism of the brain. The damage of the vessels can be demonstrated even before the appearance of clinical symptoms. B-mode ultrasound can be useful in the examination of large and medium arteries, whereas SPECT, MRI, and transcranial Doppler are able to recognize disturbances of the microcirculation. Previous studies using Doppler ultrasound found decreased reactivity and reserve capacity of large and medium arteries in both types of diabetes (4-6). Fülesdi et al. found that the decrease in circulation became pronounced $10 \mathrm{y}$ after the diagnosis of both types of diabetes $(5,6)$. Other studies used the SPECT method to measure semiquantitatively the CBF and reserve capacity and found a significant decrease in both parameters (7-9). Keymeulen et al. found an association among CBF, hemoglobin $\mathrm{A} 1 \mathrm{c}(\mathrm{HbA} 1 \mathrm{c})$, and the duration of T1DM (10). T2DM patients demonstrated damaged cognitive functions in line with the reduced circulation of the frontal and parietal lobes (11). In T1DM patients with proliferative retinopathy, SPECT and MRI examinations revealed the highest decrease in the circulation of the frontal lobe, followed by the occipital lobe $(7,12)$.

However, no previous study compared the changes detected in the circulation of the brain between T1DM and T2DM. We aimed to study the possible differences in global and regional cerebral circulation and reserve capacity in these patients.

\section{MATERIALS AND METHODS}

A total of 17 patients with T1DM (mean age $\pm \mathrm{SD}, 34.6 \pm 10.3 \mathrm{y}$; body mass index [BMI], $22.0 \pm 2.6 \mathrm{~kg} / \mathrm{m}^{2}$ ) and 43 patients with T2DM (mean age $\pm \mathrm{SD}, 54 \pm 7.8 \mathrm{y}$; BMI, $31.5 \pm 5.5 \mathrm{~kg} / \mathrm{m}^{2}$ ) were enrolled in the study. Informed consent was collected from all patients.

A dual-head $\gamma$-camera (Helix; Elscint) was used to image brain perfusion in basal and acetazolamide-challenged states on separate days. Acquisition of a dynamic image series $(70 \times 1 \mathrm{~s})$ from the anterior view, including both the brain and the aortic arch, was started immediately after the administration of $600 \mathrm{MBq}$ of ${ }^{99 \mathrm{~m}} \mathrm{Tc}-$ 
hexamethylpropylene amine oxime. The SPECT study was started after $60 \mathrm{~min}$. For the stimulation study, the radiopharmaceutical was injected $15 \mathrm{~min}$ after acetazolamide $(15 \mathrm{mg}$ of diamox per kilogram; Lederle Laboratories) because earlier studies showed maximal vasodilation at this time (13).

From the dynamic series, we estimated hemispheric CBF in $\mathrm{mL} / \mathrm{min} / 100 \mathrm{~g}$ units, using the Patlak plot (14).

Reconstructed slices were spatially normalized to the International Consortium for Brain Mapping brain SPECT template (15) using affine transform and were either scaled by dividing by the average of the whole brain or transformed to absolute $(\mathrm{mL} / \mathrm{min} /$ $100 \mathrm{~g}$ of brain tissue) units using the global CBF obtained from the dynamic study. Regional perfusion values were calculated from 8 standard brain areas (frontal, parietal, occipital, temporal, thalamus, basal ganglia, brain stem, and cingulate).

Carotid arteries were examined with Doppler ultrasound to exclude significant stenosis. Laboratory parameters (HbA1c, highsensitivity C-reactive protein, and lipid levels) were measured routinely in the central biochemical laboratory of the university. The patients were in a stable metabolic state; neither hypoglycemia nor hyperglycemia was present during the study or in the previous month. Most of the patients had hypertension, but only patients who were under effective treatment and measured normal blood pressure 3 mo before the study were involved.

The SPSS 15 statistical software package (SPSS Inc.) was used for data analysis. The Student $t$ test was applied to compare the mean values of the patient groups, with Bonferroni adjustment for multiple comparisons. In the case of laboratory parameters with nongaussian distribution, a nonparametric Kruskal-Wallis test was applied to compare the groups. To identify influencing factors, multistep regression analysis was performed.

\section{RESULTS}

Figure 1 shows the anthropometric and laboratory data of the patients.

\section{Dependence of Brain Perfusion on Type of Diabetes}

Global CBF was diminished in T2DM patients, compared with T1DM individuals, both in a resting condition $(P<0.001)$ and after acetazolamide stimulation $(P<$ 0.05 ), as shown in Figure 2. The blood flow of the examined cerebral regions-with the exception of the occipital lobe and the brain stem-also showed a significant difference between the 2 groups ( $P<0.05$; Fig. 3). Our results indicate that $\mathrm{CBF}$ is dependent on age in both groups (T1DM, $P<0.01$; T2DM, $P<0.05$ ). However, the slopes of the age-dependence trend lines are different in the 2 groups, which indicates a different effect of T1DM and T2DM on CBF ( $P<0.05$; Fig. 4). Reserve capacity, which is the increase in blood flow after acetazolamide stimula-

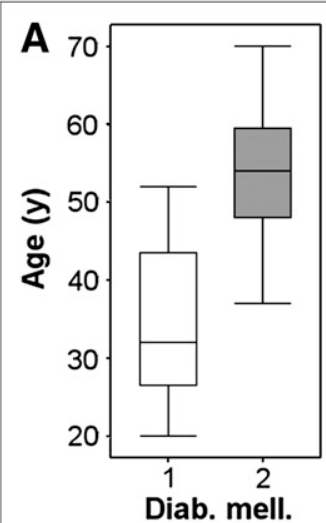

B

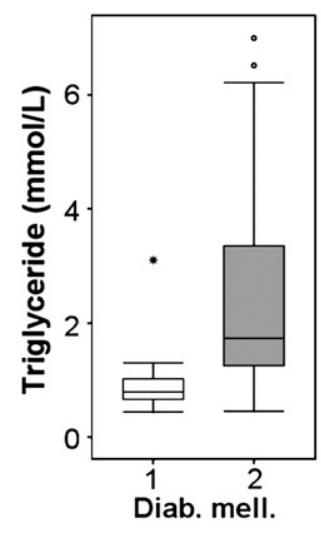

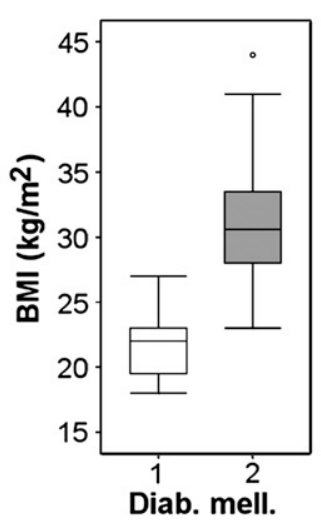

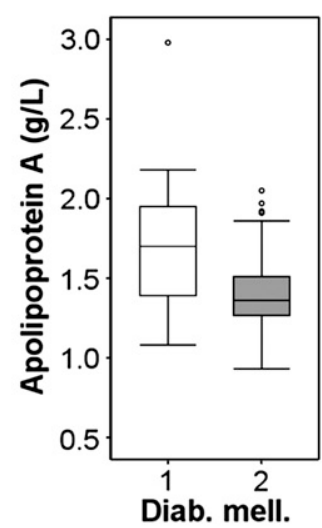

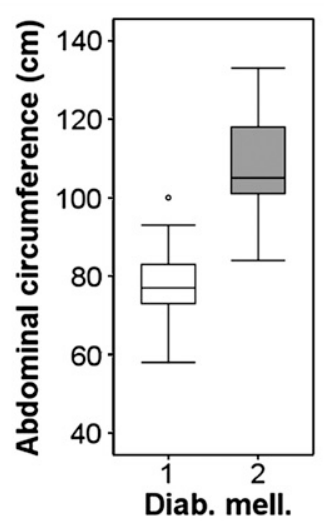
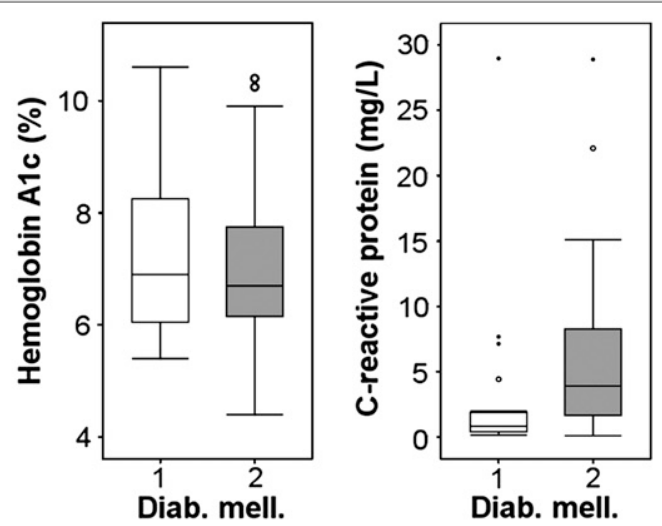

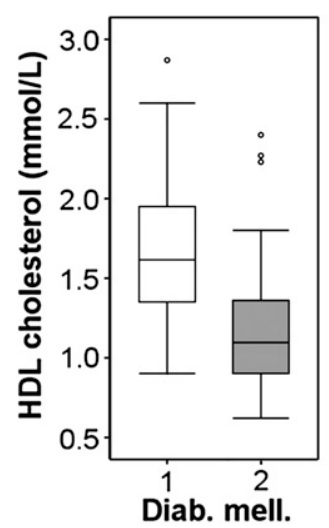

FIGURE 1. Anthropometric and laboratory data of patients. Box-and-whisker plots are shown, summarizing median, quartiles, and extreme values. Box represents interquartile range, which contains middle $50 \%$ of values. Whiskers are lines that extend from box to highest and lowest values, excluding outliers. Line across box indicates median. All differences are significant $(P<$ 0.05). Diab. mell. = diabetes mellitus. 


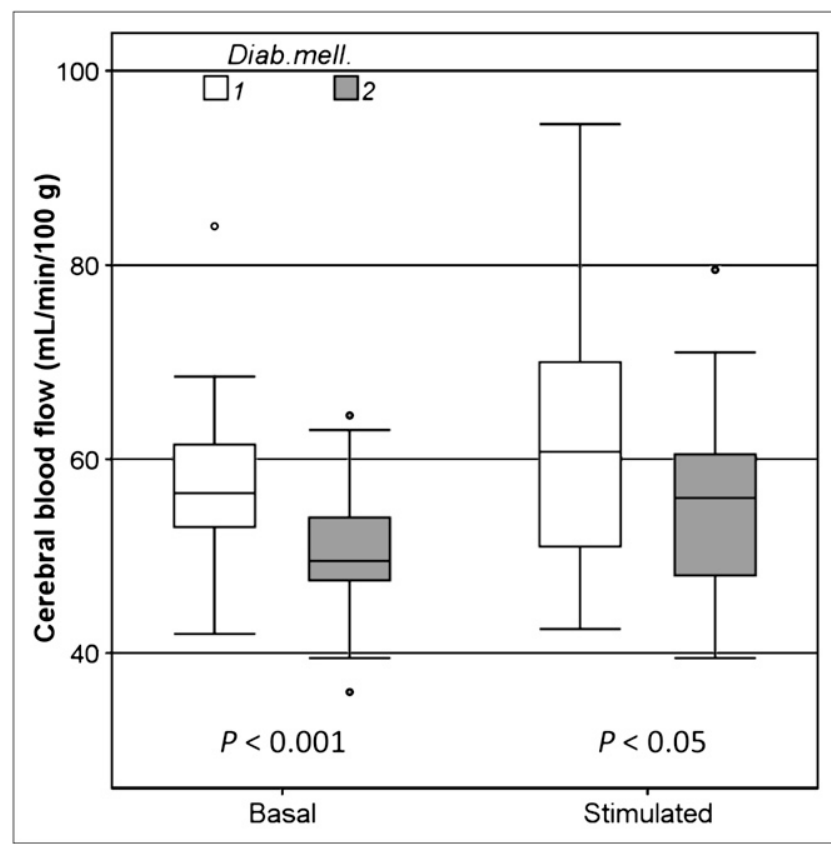

FIGURE 2. Global CBF at rest and after acetazolamide stimulation in both diabetic groups (in units of $\mathrm{mL} / \mathrm{min} / 100 \mathrm{~g}$ of tissue). Box-and-whisker plots are shown, summarizing median, quartiles, and extreme values. Box represents interquartile range, which contains middle $50 \%$ of values. Whiskers are lines that extend from box to highest and lowest values, excluding outliers. Line across box indicates median. Diab. mell. = diabetes mellitus.

tion, did not show a significant difference between the 2 groups. We could not find any association between the duration of diabetes and CBF.

\section{The Effect of Diabetes on Regional CBF}

When investigating the blood flow in each cerebral region related to the average of the whole brain, we found smaller differences between the frontal and occipital lobes in the T2DM than in the T1DM group (Fig. 5). The perfusion ratio of the frontal to the occipital lobe was significantly diminished (Fig. 6) in the T2DM group, both in resting conditions $(P<0.0005)$ and after acetazolamide stimulation $(P<0.017)$. Typical slices are shown in Figure 7.

The cerebral circulation of patients with proliferative retinopathy was slightly lower than that of patients with nonproliferative retinopathy and without retinopathy, but these differences were not significant.

\section{Factors Influencing CBF (General Linear Model)}

When investigating the diabetic groups separately, we found that in addition to the type of diabetes (interacting with age) the following factors significantly influence basal brain perfusion: BMI $(P=0.0007)$ and abdominal circumference $(P=0.004)$ in close interaction $(P=0.0008)$ and high-density lipoprotein (HDL) level $(P=0.011)$.

Among the diabetic patients, acetazolamide-stimulated brain perfusion was significantly influenced by triglyceride

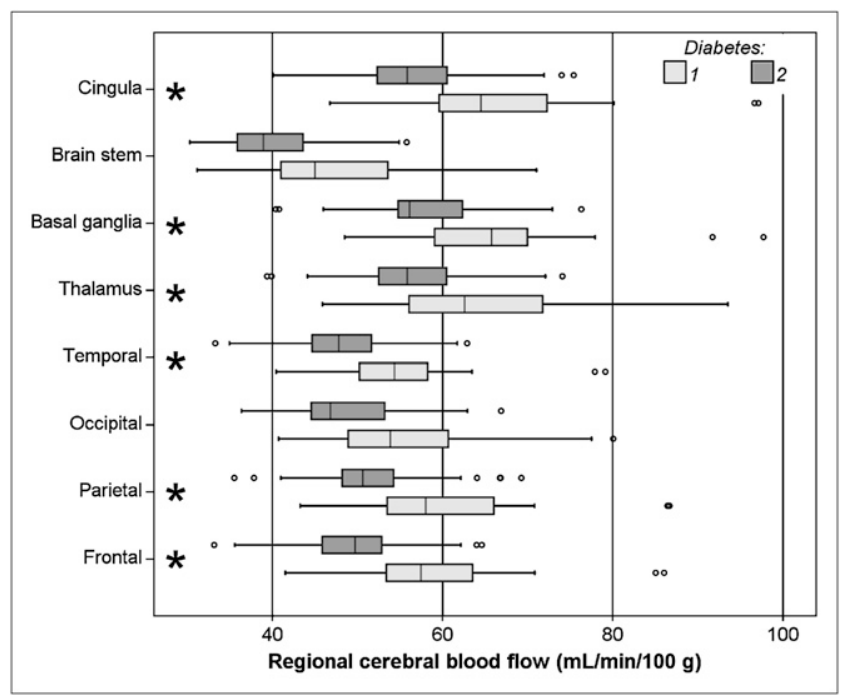

FIGURE 3. Distribution of CBF values in each region in both groups. Box-and-whisker plots are shown, summarizing median, quartiles, and extreme values. Box represents interquartile range, which contains middle $50 \%$ of values. Whiskers are lines that extend from box to highest and lowest values, excluding outliers. Line across box indicates median. ${ }^{\star} P<0.05$.

$(P=0.006)$ and HDL $(P=0.031)$, with a considerable interaction between them $(P=0.006)$.

Reserve capacity, expressed as the (absolute or relative) change in perfusion as a consequence of acetazolamide challenge, did not differ in the 2 patient groups and did not show any dependence on the investigated parameters.

There was no difference between the HbAlc levels of the 2 groups.

\section{DISCUSSION}

Diabetes mellitus damages both large and small cerebral arteries. SPECT and MRI are capable of examining global

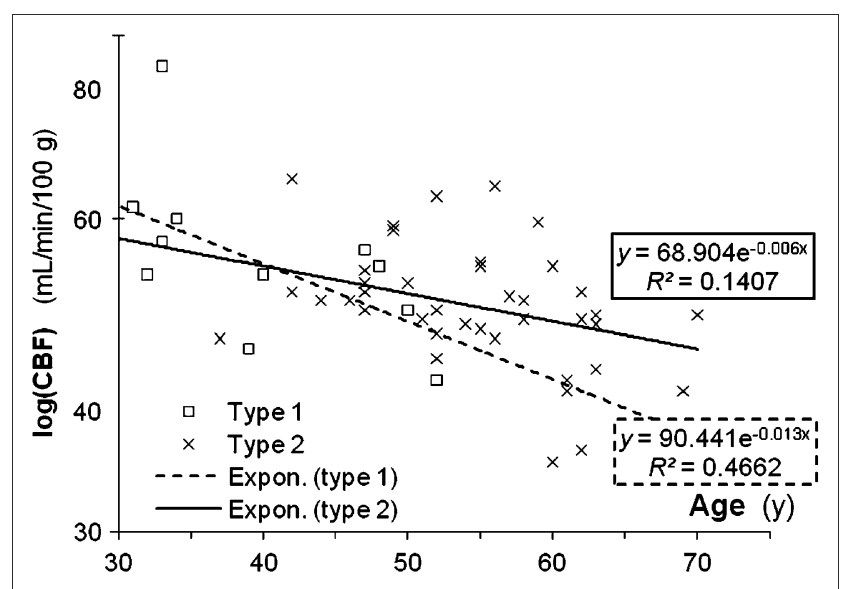

FIGURE 4. Global CBF (mL/min/100 g) as function of age in both diabetic groups. Expon. = exponential. 


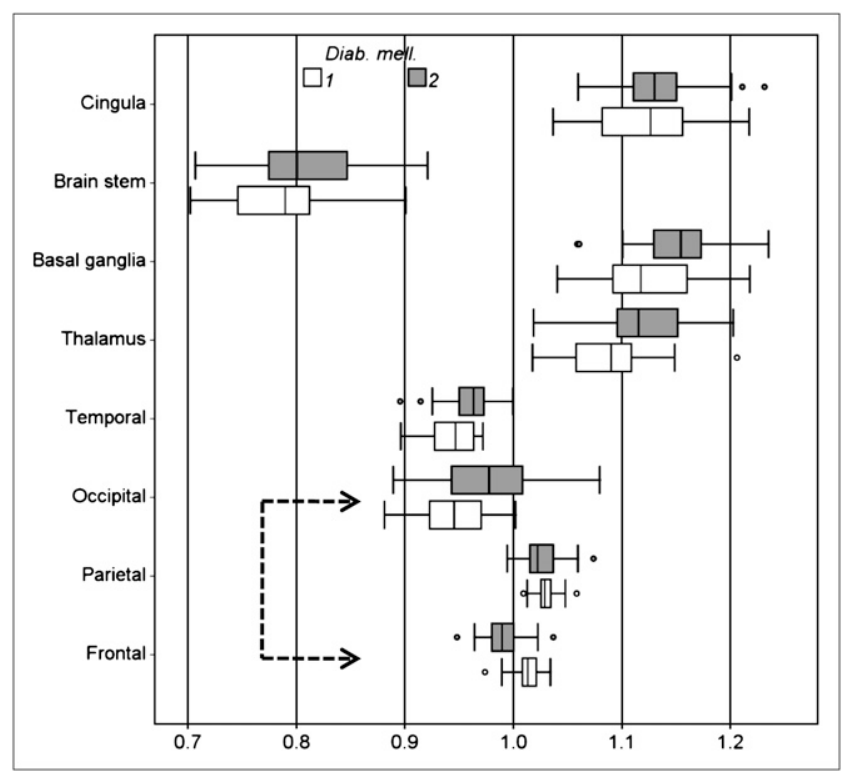

FIGURE 5. Regional CBF normalized to average of whole brain, in both diabetic groups. There was significant difference in frontal and occipital regions $(P<0.05$, indicated by arrow), in which opposite relative changes could be observed. Box-and-whisker plots are shown, summarizing median, quartiles, and extreme values. Box represents interquartile range, which contains middle $50 \%$ of values. Whiskers are lines that extend from box to highest and lowest values, excluding outliers. Line across box indicates median.

CBF. Previous studies using mostly semiquantitative methods to compare regional cerebral flow with the global brain circulation in diabetic patients or with the same cerebral regions of healthy controls detected decreased CBF and reserve capacity in diabetic patients $(9,10)$. In contrast to these studies, absolute values of brain circulation were examined in our study $(\mathrm{mL} / \mathrm{min} / 100 \mathrm{~g}$ of brain tissue).

Examinations were performed in 2 steps: in a resting position and after acetazolamide stimulation. Acetazolamide is a reversible inhibitor of carbonic anhydrase. After acetazolamide administration, the extracellular partial pressure of $\mathrm{CO}_{2}$ increases and $\mathrm{pH}$ decreases, resulting in a dosedependent vasodilation of the microvessels. Therefore, an increased amount of blood is flowing through these dilated resistance arterioles (13).

In our study regarding the global and the regional brain blood flow, both basal and acetazolamide-stimulated circulation was lower in T2DM patients than in T1DM individuals. For ethical reasons, we have not completed basal and acetazolamide-stimulated study pairs for healthy controls. However, applying the same method for calculating the global CBF in absolute units, we found previously (16) that a group of 12 healthy control subjects had a mean \pm SD of $68.5 \pm 9.7 \mathrm{~mL} / \mathrm{min} / 100 \mathrm{~g}$ global CBF after acetazolamide stimulation, higher than that in any of the diabetic groups

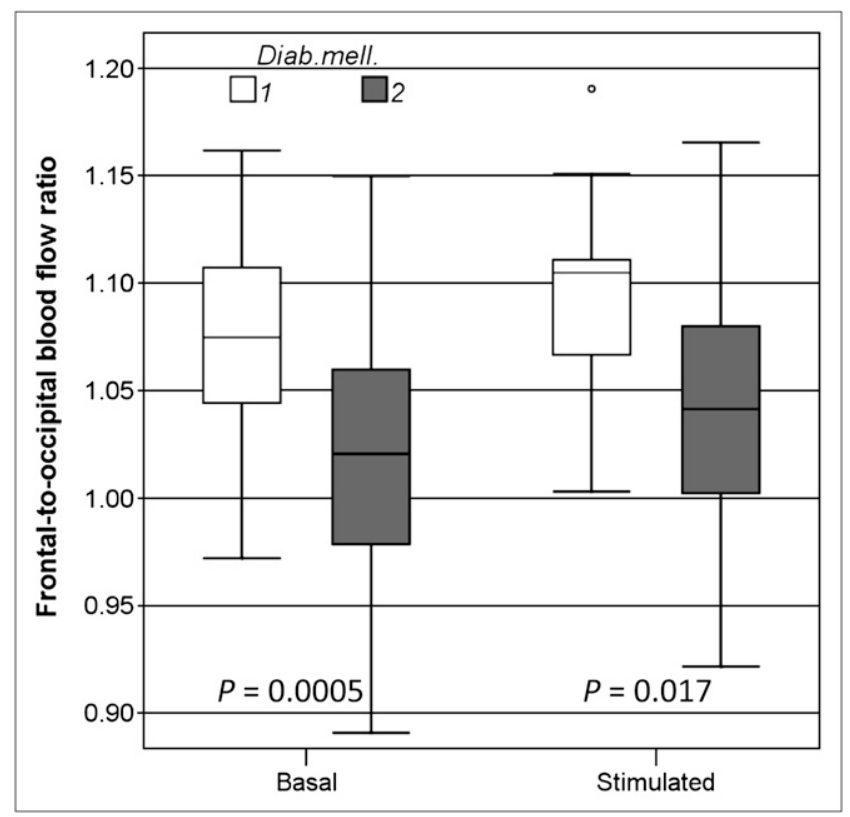

FIGURE 6. Ratio of perfusion of frontal over occipital lobe was significantly different in diabetic groups in both resting state and after acetazolamide stimulation. Box-and-whisker plots are shown, summarizing median, quartiles, and extreme values. Box represents interquartile range, which contains middle $50 \%$ of values. Whiskers are lines that extend from box to highest and lowest values, excluding outliers. Line across box indicates median. Diab. mell. = diabetes mellitus.

of the present study. This confirms the tendency that CBF is decreased in both diabetic states. The difference was significant only for the T2DM group with Dunnett $t$ test.

The reduction in brain circulation, however, can be explained - at least partially — by the difference of the mean age between the 2 groups (T2DM patients were generally older), because cerebral circulation decreases by age (17). This may be a potential limitation to the interpretation of our results; however, the slopes of the age-dependency trend lines of blood flow are different in the 2 groups, which means that T1DM and T2DM affect CBF differently.

Examining and comparing the relative blood flow of each brain region, it is an interesting and new result that the ratios of the frontal and the occipital lobe circulation were significantly diminished in T2DM patients both in a resting position and after acetazolamide stimulation. This ratio is independent of age, meaning that the circulation of the frontal lobe is damaged more considerably in T2DM patients.

Compared with other lobes, earlier and greater reductions in blood flow of the frontal and parietal lobes of diabetic patients were reported, together with a decrease in cognitive functions (9); however, studies comparing CBF of T1DM and T2DM patients were not performed before.

In addition to diabetes, hypertension and hyperlipidemia were reported to have a considerable impact on cerebral 

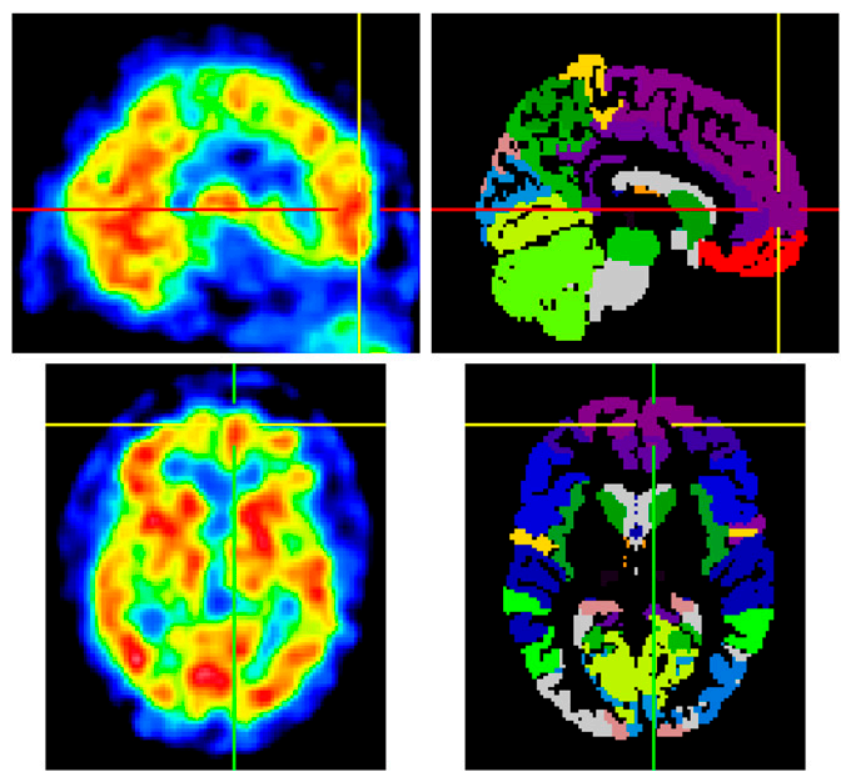

FIGURE 7. Sagittal and transversal SPECT slices of a T2DM patient (left) with frontal-to-occipital ratio of 0.915 . Corresponding slices of International Consortium for Brain Mapping template are shown on right.

blood circulation $(9,18)$. CBF is affected primarily by systolic blood pressure. Hypertension was present in many of our subjects, but all patients were treated adequately and had normal blood pressure. The effective treatment of hypertension gradually normalizes the autoregulation of cerebral vessels, and the results of vasoreactive tests do not differ from those of patients without hypertension (19).

Using the SPECT method, Wakisaka et al. found that CBF correlated inversely with total cholesterol and also showed a positive correlation with HDL cholesterol (9). In our study, we found a positive association between basal circulation and HDL cholesterol, whereas acetazolamidestimulated blood flow had a significant positive association with HDL cholesterol and it correlated negatively with triglyceride. These data agree with the atherogenic dyslipidemia found previously in diabetic patients but conflict with the results of Kerényi et al., who could not detect any difference in the reserve capacity between hyperlipidemic patients and healthy subjects using transcranial Doppler ultrasound (20). It is a possible explanation that the patients participating in the latter study had isolated hypercholesterolemia and no diabetes, an only slightly elevated triglyceride level, and a normal HDL level. In contrast, our patients had multiple risk factors.

Regarding the reserve capacity, which represents the reactivity of the cerebral arteries, we did not find a significant difference between the 2 groups.

We did not find any association between CBF and $\mathrm{HbA1c}$ level. Previous data are conflicting in this respect. Keymeulen et al. could prove this association in T1DM patients (10), whereas Fülesdi et al. did not find a correlation between these parameters either in T1DM or in T2DM patients $(5,6)$. The reduction in $\mathrm{CBF}$ is probably due to the continuously elevated glucose level through years; in contrast, a change in HbAlc level is seen an average of only $8-10 \mathrm{wk}$ before blood sampling.

The strong association of CBF with BMI is an important finding concerning mainly T2DM patients. Obesity is related closely to insulin resistance and T2DM and probably affects the vascular system through the same pathways.

A considerable result of the present study is that the 2 types of diabetes affect the regional CBF differently; namely, the decrease of the frontal circulation is relatively greater in T2DM patients.

Examining T1DM patients with MRI, Wessels et al. found decreased frontal lobe circulation in subjects with proliferative retinopathy in contrast to those without retinopathy or healthy subjects (12). Quirce et al. also examined T1DM patients and came to similar conclusions (7). In our study, cerebral circulation was decreased in patients with proliferative retinopathy, compared with those with nonproliferative retinopathy and without retinopathy. However, the difference was not significant, probably because of the small number of the individuals.

\section{CONCLUSION}

T1DM and T2DM affect differently the CBF. In T2DM patients, the circulation of the frontal lobe is damaged primarily. Basal brain perfusion is influenced by age, BMI, and HDL, whereas acetazolamide-stimulated circulation showed a correlation with triglyceride and HDL. These data call the attention to the necessity of a "holistic view" of treatment, including lifestyle modifications to preserve $\mathrm{CBF}$ and reduce disease progression.

\section{ACKNOWLEDGMENT}

This study was partially financed by the Mecenatura grant of the University of Debrecen.

\section{REFERENCES}

1. Wilson PW, Bozeman SR, Burton TM, Hoaglin DC, Ben-Joseph R, Pashos CL Prediction of first events of coronary heart disease and stroke with consideration of adiposity. Circulation. 2008;118:124-130.

2. Viberti G. The need for tighter control of cardiovascular risk factors in diabetic patients. J Hypertens Suppl. 2003;21:S3-S6

3. Novak V, Last D, Alsop DC, et al. Cerebral blood flow velocity and periventricular white matter hyperintensities in type 2 diabetes mellitus. Diab Care. 2006;29:1529-1534.

4. Hidasi E, Káplár M, Diószeghy P, et al. No correlation between impairment of cerebrovascular reserve capacity and electrophysiologically assessed severity of neuropathy in noninsulin-dependent diabetes mellitus. J Diab Comp. 2002;6: 228-234.

5. Fülesdi B, Limburg M, Bereczki D, et al. Impairment of cerebrovascular reactivity in long-term type 1 diabetes. Diabetes. 1997;46:1840-1845.

6. Fülesdi B, Limburg M, Bereczki D, et al. Cerebrovascular reactivity and reserve capacity in type II diabetes mellitus. J Diab Comp. 1999;13:191-199.

7. Quirce R, Carril JM, Jiménez-Bonilla JF, et al. Semi-quantitative assessment of cerebral blood flow with ${ }^{99 \mathrm{~m}} \mathrm{Tc}$-HMPAO SPET in type I diabetic patients with no clinical history of cerebrovascular disease. Eur J Nucl Med. 1997;24:15071513 
8. Carril JM, Quirce R, Amado JA, et al. Perfusion SPECT with ${ }^{99 m} \mathrm{Tc}-\mathrm{HMPAO}$ in type I diabetics with no background of central neurologic symptoms: a study of activation with acetazolamide. Rev Esp Med Nucl. 2000;19:187-191.

9. Wakisaka M, Nagamachi S, Inoue K, Morotomi Y, Nunoi K, Fujishima M. Reduced regional cerebral blood flow in aged noninsulin-dependent diabetic patients with no history of cerebrovascular disease: evaluation by $N$-isopropyl- ${ }^{123} \mathrm{I}-p$-iodoamphetamine with single-photon emission computed tomography. J Diab Comp. 1990;4:170-174.

10. Keymeulen B, de Metz K, Cluydts R, Bossuyt A, Somers G. Technetium-99m hexamethylpropylene amine oxime single-photon emission tomography of regional cerebral blood flow in insulin-dependent diabetes. Eur J Nucl Med. 1996;23:163-168.

11. Niwa H, Koumoto C, Shiga T, et al. Clinical analysis of cognitive function in diabetic patients by MMSE and SPECT. Diabetes Res Clin Pract. 2006;72:142-147.

12. Wessels AM, Simsek S, Remijnse PL, et al. Voxel-based morphometry demonstrates reduced grey matter density on brain MRI in patients with diabetic retinopathy. Diabetologia. 2006;49:2474-2480.

13. Settakis G, Molnár C, Kerényi L, et al. Acetazolamid as a vasodilatory stimulus in cerebrovascular diseases and in conditions affecting the cerebral vasculature. Eur J Neurol. 2003;10:609-620.
14. Matsuda H, Tsuji S, Shuke N, Sumiya H, Tonami N, Hisada K. A quantitative approach to technetium-99m hexamethylpropylene amine oxime. Eur J Nucl Med. 1992;19:195-200.

15. Mazziotta J, Toga A, Evans A, et al. A probabilistic atlas and reference system for the human brain: International Consortium for Brain Mapping (ICBM). Philos Trans R Soc Lond B Biol Sci. 2001;356:1293-1322.

16. Garai I, Varga J, Szomják E, et al. Quantitative assessment of blood flow reserve using ${ }^{99 m}$ Tc-HMPAO in carotid stenosis. Eur J Nucl Med Mol Imaging. 2002;29: 216-220.

17. Stoquart-ElSankari S, Baledent O, Gondry-Jouet C, Makki M, Godefroy O, Meyer ME. Aging effects on cerebral blood and cerebrospinal fluid flows. J Cereb Blood Flow Metab. 2007;27:1563-1572.

18. Ficzere A, Valikovics A, Fülesdi B, Juhász A, Czuriga I, Csiba L. Cerebrovascular reactivity in hypertensive patients: a transcranial doppler study. J Clin Ultrasound. 1997;25:383-389.

19. Pieniazek W, Dimitrow PP, Jasinski T. Comparison of the effect of perindopril and acebutolol on cerebral hemodynamics in hypertensive patients. Cardiovasc Drugs Ther. 2001;15:63-67.

20. Kerenyi L, Fulesdi B, Ficzere A, et al. Cerebrovascular reserve capacity in patients with hyperlipidaemia. J Clin Ultrasound. 2000;28:115-121. 